\title{
Transfusion Support of Minority Patients: Extended Antigen Donor Typing and Recruitment of Minority Blood Donors
}

\author{
Jenna Khan ${ }^{a}$ Meghan Delaney ${ }^{b, c}$ \\ a Department of Laboratory Medicine, University of Washington, Seattle, WA, USA; \\ bPathology \& Laboratory Medicine Division, Children's National Health System, Washington, DC, USA; \\ ${ }^{c}$ Department of Pathology \& Pediatrics, The George Washington University, Washington, DC, USA
}

\section{Keywords}

Allosensitization · Antigens · Blood groups · Blood transfusion

\section{Summary}

One of the most important and persistent complications of blood transfusion is red blood cell (RBC) alloimmunization. When a patient is exposed to RBC antigens that differ from their own they can form alloantibodies to these foreign antigens. Blood group antigens are highly conserved and follow ancestral patterns of inheritance that may demonstrate population restriction. Minority populations who require chronic transfusion are at particularly high risk of alloimmunization when the blood donor population does not share the same ancestral background, resulting in exposure to non-self RBC antigens. It is incumbent on blood collectors to support patients with risk factors for alloimmunization as well as patients who have already formed alloantibodies. Increasing utilization of RBC genotyping may represent an opportunity to improve access to RBC units from donors that match the extended RBC phenotype of all possible patients.

(C) 2018 S. Karger GmbH, Freiburg

\section{Introduction}

Red blood cell (RBC) transfusions are a critical part of patient care. Some patients may require antigen-matched RBCs for transfusion. The ancestral genetic background of a patient can impact accessibility of antigen-matched blood RBC products when the patient does not share ancestry with the majority of blood donors in the population $[1,2]$. For example, RBC transfusion is a key component in the treatment of patients with sickle cell disease (SCD). The number of patients with SCD requiring acute and/or chronic $\mathrm{RBC}$ transfusion continues to rise [3]. In the US, the ancestral background of patients with SCD is usually of African or Mediterranean descent, whereas the donor population is predominantly Caucasian, increasing patient risk for alloimmunization. Conversely when patients with SCD receive transfusions in regions where the blood donors share ancestral backgrounds, the rate of alloimmunization may be less [4]. For the purposes of this review, we will use 'minority' population to refer to the situation described above.

Although patients with SCD present the largest documented experience of how chronic transfusion with patient/donor antigen mismatches can lead to medical consequences, any patient with population-restricted blood group phenotypes is at risk for alloimmunization and its consequences. To prevent alloimmunization and to find compatible units after alloimmunization, an adequate supply of antigen-negative units needs to be maintained. The challenge falls to blood collectors to recruit donors, to use cost-effective testing approaches to type the inventory, and to carry out efficient inventory management.

\section{Consequences of Alloimmunization}

Depending on the antigen and clinical significance of the antibody formed, patients can suffer morbidity and mortality due to an acute or delayed hemolytic transfusion reaction (DHTR) if incompatible blood products are transfused. It is estimated that $4-7 \%$ of transfused SCD patients develop an overt DHTR, with an even

\section{KARGER}

() 2018 S. Karger GmbH, Freiburg

Fax +497614520714 
higher incidence of mild DHTR likely being underreported. Approximately $30 \%$ of unexpected $\mathrm{Rh}$ antibodies in patients with SCD demonstrate associated decreased RBC survival [5].

A rare but life-threatening consequence of recurrent transfusions is a hyperhemolytic reaction. This reaction typically occurs in patients with hemoglobinopathies and has been reported in 1-19\% of transfusions in patients with SCD [6]. Although mechanistically not well understood, it is likely a complication of alloimmunization, with possible contribution of an underlying genetic predisposition $[7,8]$.

The development of RBC alloantibodies also impacts patient care by increasing the cost and time required to find compatible $\mathrm{RBC}$ units. Once an RBC antibody is identified, all subsequent transfusions must be negative for that antigen to prevent a DHTR from a robust secondary immune response. An additional risk for previously sensitized patients is the inability to detect evanesced RBC antibodies at future transfusion events; $18-64 \%$ of all antibodies (depending on the antibody detection technique used and the time since initial discovery) and $>80 \%$ of anti-D in $\mathrm{D}+$ patients become undetectable over time $[5,6,9,10]$. Despite frequently becoming undetectable, anti-D in serologically $\mathrm{D}+$ patients remains clinically significant, and overt hemolytic transfusion reactions have been reported in patients with SCD [5]. Failure to identify pre-existing antibodies is a significant contributor to hemolytic transfusion reaction fatalities reported to the US Food and Drug Administration (FDA) each year [11]. How to best facilitate communication of the clinical significance of previously recognized antibodies to patients and physicians remains to be determined, but is an important area of consideration to avoid incompatible blood transfusions, particularly when patient care occurs at multiple hospitals and records of historical antibodies may not be readily available at the time of transfusion.

Minority patients may be at greater risk of complications from alloimmunization because the presence of antibodies may not be accurately characterized. One reason is that they are more likely to be negative for high-prevalence antigens. Antibodies to high-prevalence RBC antigens will react with all reagent RBCs. This is further complicated if the patient also has a positive direct antiglobulin test (DAT), as patients with SCD frequently do; the antibody to a high-prevalence antigen can then easily be confused with a warm autoantibody. Clinically significant high-prevalence antigens include $\mathrm{U}$ (about $2 \%$ of African Americans are $\mathrm{U}$ - or $\mathrm{U}$ variant), Js $\mathrm{s}^{\mathrm{b}}$, $\mathrm{Jo}^{\mathrm{a}}$, or Hy (about $1 \%$ of African Americans are Js(b-), Jo(a-), or Hy-) [12].

In addition, because most reagent RBCs are not from minority populations, there is a risk that immunogenic $\mathrm{Rh}$ variants and other low-prevalence antigens are not expressed on the reagent $\mathrm{RBCs}$, rendering antibody detection tests false-negative. When an antibody screen is negative, it is common practice to perform a computer match rather than a physical testing match, increasing the risk of a hemolytic transfusion reaction. Examples of immunogenic antigens that may fall into this category include $\mathrm{Rh}$ variants (about $20 \%$ of African Americans have the partial Rh antigens V/ VS, Go ${ }^{\mathrm{a}}$, or DAK [13]), Dombrock (Do $\left.{ }^{\mathrm{a} / \mathrm{b}}, \mathrm{Hy}, \mathrm{Jo}^{\mathrm{a}}\right)$, other Kell sys- tem antigens $\left(\mathrm{Kp}^{\mathrm{a} / \mathrm{b}}, \mathrm{Js}^{\mathrm{a} / \mathrm{b}}\right)$, Colton $\left(\mathrm{Co}^{\mathrm{a} / \mathrm{b}}\right), \mathrm{Yt}^{\mathrm{a} / \mathrm{b}}$, Lutheran $\left(\mathrm{Lu}^{\mathrm{a} / \mathrm{b}}\right)$, Diego $\left(\mathrm{Di}^{\mathrm{a} / \mathrm{b}}\right)$, and Scianna $(\mathrm{Sc} 1 / 2)$. Antibodies to Dombrock antigens are particularly troublesome because they are very difficult to detect in serologic testing despite being able to cause severe in vivo hemolysis. These antibodies are weakly reactive in multi-specific sera or undetectable on antibody screen and often have a negative DAT due to weak avidity. There are no commercially available antisera to type patient or donor cells for Dombrock antigens making genotyping the only method to identify patients at risk and find compatible units [12]. When there is compromised survival of transfused RBCs in minority patients, there needs to be a high suspicion for alloimmunization to a non-serologically detectable antibody; incompatibility due to variant Rh and Dombrock antigens should always be ruled out [12]. In these cases, genotyping can be particularly useful to clarify antibody specificity, identify if there is a lack of a high-prevalence antigen, and identify appropriate donors.

Another important complication of alloimmunization is the long-recognized phenomenon that after patients develop one RBC antibody, they are more likely to develop additional antibodies the so-called immune responder' phenotype [14]. Although the exact cause of an immune responder phenotype is not well understood, candidate genes and gene polymorphisms have been described $[15,16]$.

In addition, mouse models have demonstrated that priming of $\mathrm{CD} 4+\mathrm{T}$ cells with one $\mathrm{RBC}$ antigen $(\mathrm{K})$ may promote a humoral immune response to disparate $\mathrm{RBC}$ antigens when both antigens are present on the same RBC [14]. These results suggest that the prevention of an initial alloimmunization event may be even more important than previously appreciated to prevent the development of subsequent antibodies. For patients with a tendency toward forming RBC antibodies, and also having a RBC phenotype with either multiple negative antigens and/or lacking high-prevalence antigens, compatible units may become so rare as to make transfusion support virtually impossible [17].

\section{RBC Antigen Disparity and Transfusion Matching}

The incidence of alloimmunization is highly variable between individual patients; all of the underlying mechanisms driving these differences remain to be fully elucidated [18]. Patients who develop antibodies tend to have received significantly more transfusions, making alloimmunization a particular problem for patients requiring chronic RBC transfusion support $[5,19]$. Even within chronically transfused populations, there is a notable discrepancy in the incidence of antibody formation. Patients with SCD have alloimmunization rates of up to $36 \%$ compared to $5 \%$ in transfusion-dependent thalassemia and $2 \%$ in other regularly transfused patient populations [13]. One major difference between these populations is that SCD occurs predominantly in people of African ancestry, whereas the donor population in North America and Europe is predominantly of European ancestry. As the fundamental requirement for alloimmunization to occur is exposure to a foreign anti- 
Table 1. Frequency of negative antigens in SCD patients [17]

\begin{tabular}{lcc}
\hline $\begin{array}{l}\text { Number of RBC antigens } \\
\text { that are lacking }\end{array}$ & Patients $(\mathrm{n}=70)$ & Prevalence (\%) \\
\hline 3 & 1 & 1.4 \\
4 & 12 & 17.1 \\
5 & 14 & 20.0 \\
6 & 29 & 41.4 \\
7 & 14 & 20.0 \\
\hline
\end{tabular}

gen, the difference in prevalence between African Americans and Caucasians of several RBC antigens, as well as a lack of certain high-prevalence antigens in populations of African descent, is likely a major contributor to the higher incidence of alloimmunization [20]. For instance, only about $2 \%$ of the Caucasian population is negative for the $\mathrm{C}, \mathrm{K}, \mathrm{E}, \mathrm{Fy}^{\mathrm{a}}$, and $\mathrm{S}$ antigens, and extended matching for $\mathrm{D}, \mathrm{C}, \mathrm{c}, \mathrm{E}, \mathrm{e}, \mathrm{K}, \mathrm{S}, \mathrm{Fy}^{\mathrm{a}}$, and $\mathrm{Jk}^{\mathrm{b}}$ is predicted to be present in only $0.6 \%$ of Caucasian donors compared to $14.6 \%$ of African American donors [18, 20]. Because the number of negative antigens is high in patients with SCD, the likelihood that they will be exposed to non-self antigens is potentially greater (table 1) [17].

The diversity of the Rh blood group system in people of African descent can also provide a basis for disparity for patients with SCD who are chronically transfused. Over 500 RHD and over 140 RHCE allelic variations have been reported [21]; $90 \%$ of SCD patients and healthy populations of African ancestry carry at least 1 variant RHD or RHCE allele $[5,13]$. The term 'variant' will be used here to mean the lack of a conventional allele (i.e. homozygous or compound heterozygous for variant alleles). It encompasses alleles that code for weak D and partial D expression, as these classifications are not always precisely known since most $R H D$ variants have not been adequately characterized for potential immunogenicity, particularly when considering transfusion with other Rh variants [22, 23]. In addition, $\mathrm{Rh}$ antigen specificities are particularly complex because variants in either RHD or RHCE genes can express D-like, C-like, or E-like antigens, thus the immunogenic potential of one gene should not be evaluated in isolation $[12,24]$.

The phenotype of $\mathrm{Rh}$ variants cannot be reliably detected by routine serologic testing and in some cases will even have misleading serological results that can lead to inaccurate phenotyping results. Approximately $20 \%$ of patients with SCD that phenotype as $\mathrm{C}+$ actually express a variant $\mathrm{C}$ due to the $\mathrm{C}$ antigen being encoded by a hybrid $R H D^{\star} D I I I a-C E(4-7)-D$. $30 \%$ of patients with this hybrid gene (in the absence of a conventional $R H C E^{*} C e$ allele in trans) made anti- $\mathrm{C}$ after repeated exposure to conventional $\mathrm{C}+$ $\mathrm{RBCs}$, suggesting that they should be treated as $\mathrm{C}$ - for transfusion purposes [12]. The e antigen in patients with SCD is also not adequately evaluated with routine serology. Although nearly all SCD patients of African descent will be e+, approximately one-third will be homozygous for a partial or altered (variant) e antigen and are capable of making an anti-e alloantibody [12]. Only $2 \%$ of donors are $\mathrm{E}+\mathrm{e}-$, which can make finding compatible units difficult if there are additional antibodies present. Knowledge of the genotypes of both patients and donors has led to a greater understand- ing of potential mechanisms for persistent alloimmunization despite serologic antigen matching for transfusion.

In chronically transfused patients with SCD, over two-thirds of the alloantibodies formed have Rh blood group (primarily D, C, and E) or Kell (typically K) specificities [25]. This has led to evidence-based recommendations for patients with SCD to receive RBC transfusions prophylactically matched for D, C, E, and K antigens [26]. This strategy when adopted has been shown to significantly reduce alloimmunization rates from 27 to $75 \%$ with $\mathrm{ABO}-\mathrm{D}$ matching alone to 5-14\% with limited C, E, and K matching [27]. Extended matching to include the Duffy, Kidd, and MNS systems has been shown to reduce the rate of alloimmunization to $0-7 \%$ [27]. Although these approaches result in an overall reduction in alloantibody formation, clinically significant alloimmunization continues to occur [5].

A concern for transfusion of patients with SCD with extended phenotype-matched RBC units from a predominantly African descent donor population is the risk for development of antibodies to low-frequency antigens that are relatively more prevalent in populations of African descent, such as V, VS, and Js ${ }^{\mathrm{a}}[4,28]$. Js ${ }^{\mathrm{a}}$ for example occurs in $20 \%$ of African Americans compared to $0.01 \%$ of Caucasians. In a prospective study looking at the influence of minor antigen mismatches on the frequency of alloimmunization in patients with SCD, a high frequency of mismatches per transfusion episode for S (43.9\%), Do ${ }^{\mathrm{a}}(43.9 \%), \mathrm{Fy}^{\mathrm{a}}(29.2 \%), \mathrm{M}(28.4 \%)$, $\mathrm{Jk}^{\mathrm{b}}(28.1 \%), \mathrm{N}(24.0 \%), \mathrm{V}(19.3 \%)$, VS (17.9\%), and Js ${ }^{\mathrm{a}}$ (13.3\%) was noted. Of these antigens, only 3 anti-Js ${ }^{\mathrm{a}}$ antibodies developed in the 12-month study period. These antibodies all occurred in patients with higher relative exposure to $\mathrm{Js}^{\mathrm{a}}$ (increased overall frequency and frequency immediately prior to antibody development ( 3 of 4 prior transfusions)) and in patients with previous antibody formation that were receiving units antigen-negative for significant antibodies and with extended serologic matching for D, C/c, E/e, $\mathrm{K}, \mathrm{Fy}^{\mathrm{a}}$, and $\mathrm{Jk}^{\mathrm{b}}$. Given that only antibodies to Js ${ }^{\mathrm{a}}$ developed despite much higher rates of mismatch in other antigens suggests that further evaluation of the benefit of matching for $\mathrm{Js}^{\mathrm{a}}$ may be warranted. Although Js(a-) RBC units are reasonably common ( about $91 \%$ of donor units), finding antigen-negative units requires RBC genotyping methods.

Some studies have shown that transfused patients with SCD receiving units from donors with shared ethnic background may be protective. For instance, lower rates of alloimmunization have been seen in Ugandan and Jamaican patients with SCD where the donor and patient populations are ethnically more similar $[4,13,29,30]$. In theory this should reduce alloimmunization by improving the antigen discrepancy between donor and recipient and thus reducing exposure to foreign antigens [18]. However, a 15-year retrospective review of 182 patients with SCD in the US who were transfused with this strategy demonstrated alloimmunization in 71 (58\%) of chronic and 9 (15\%) of episodically transfused patients. There was a reduced rate of anti-Jk, anti-Fy, and anti-S and no cases of anti-K formation [5]. Most notably and unexpectedly, nearly two-thirds $(64.4 \%)$ of all antibodies formed had specificity for common $\mathrm{Rh}(\mathrm{D}, \mathrm{C}, \mathrm{E}, \mathrm{e})$ despite prophylactic $\mathrm{Rh}$ antigen 
matching. Only 3 cases of anti-E development could be explained by transfusion of E+ units to E- recipients due to the presence of an anti-e. The remaining antibodies to Rh antigens were unexplained, meaning that they should not have occurred because the patient was either not exposed to the corresponding foreign antigen or should not have perceived the antigen as foreign. $62 \%$ of these antibodies occurred in patients who typed serologically positive for the antigen for which they had developed an antibody and the remaining occurred in antigen-negative patients receiving corresponding antigen-negative units. Follow-up high-resolution genotyping of all patients revealed a nonconventional/variant $R H$ allele in $87 \%$ of patients. It is likely that the African American donors had a similar degree of $R H$ heterogeneity, but genotypes of all transfused units were not available for confirmation. The presence of variant or conventional alleles in transfusion recipients did not consistently correlate with the development of antibodies, suggesting that both the recipient and donor genotypes contributed to alloimmunization. This was most pronounced for RHD where only one quarter of $\mathrm{D}+$ patients with anti-D had only variant $R H D$ alleles, and of all the $\mathrm{D}+$ patients with only variant $R H D$ just $17 \%$ developed anti-D antibodies. The presence of 1 conventional $R H$ allele also did not appear to be protective against alloimmunization as just conventional e alleles was the only $R H$ genotype that did not demonstrate development of an unexpected anti-e. It is clear that serologic phenotyping is inadequate to capture allelic diversity in minority populations.

Without accurate characterization of the patient and donor genotypes, true antigen matching to prevent alloimmunization is not possible. In addition, there is still an inadequate understanding of the risk of alloimmunization with specific blood group gene haplotypes, particularly for RHD. Large, multi-institutional studies with genotyping of both patient and donor and better characterization of the specificity of antibodies formed are needed to clarify the clinical significance and immunogenic risks of variant alleles.

\section{Donor Recruitment and Antigen Typing}

It is incumbent on blood donor centers to have an adequate supply of blood products for all patients, including all patients with RBC antibodies and minority populations. For patients with SCD in particular, the goal is to consistently have enough multiple antigen-negative compatible units for prophylactic matching programs and for alloimmunized patients if an emergency large-volume transfusion is required for a stroke or other SCD-related crisis.

To maintain an adequate inventory, mass-scale RBC genotyping has been successfully implemented to replace serological screening at several donor centers $[31,32]$. The RBC genotyping approach allows for high throughput platforms. The platforms commonly used include many antigens, some with no commercially available antisera, increasing the chance of finding phenotypes that are difficult to type with serologic approaches alone. Different strategies to gain efficiency and cost-effectiveness for antigen typing programs include targeting those donors that are non-Caucasian de- scent, repeat group O donors, or those that carry variant phenotypes suggestive of more rare genotypes, such $\mathrm{as}^{\mathrm{B}} \mathrm{B}^{\mathrm{B}}$ negative [23, 31,33 . Given the improvement in, and increased availability of, high-throughput RBC genotype testing platforms, implementation at donor centers has become standard practice for many large collectors. This will likely benefit patients as the larger the available antigen-negative inventory, the more likely appropriate units can always be available. Further, action by regulatory bodies to make it easier to put historically typed RBC antigens on the RBC unit label itself can help to streamline communication between blood collector and hospital transfusion service and expedite availability of antigen-negative units.

Even as RBC genotyping programs are being implemented by many blood collectors, finding adequate units can still be a challenge, particularly when donors of similar ancestral background are the best source of RBCs for patients that require extended matching. Of annual blood donors in the US, the authors found that African Americans represent only 5\% of donors, compared with approximately $74 \%$ that were Caucasian [2]. The discrepancy is even greater for repeat donors who are predominantly older white donors (about $83 \%$ of repeat donors are white) [1]. The reasons for the lower donation rates in minority populations are multi-factorial and include increased donor ineligibility, donor deferral, and barriers to donation, particularly fear and distrust [34]. In order to increase donations, minority recruitment programs have been implemented. Unfortunately, these programs have been unsuccessful in substantially increasing repeat donations from minority populations to adequately match the needs of an increasingly diversifying population [2]. Social media campaigns for organ donation have been successful in increasing donations and could represent an underutilized strategy for donor centers to recruit younger, more diverse donors and convert them to repeat donors [35]. Some blood collectors do have active programs for minority donor recruitment; others work with raising awareness with SCD patient advocacy groups. Most strategies have had limited long-term success and require sustained efforts at significantly increased expense $[34,36]$. Given the public fascination with genetic testing and ancestry analysis, the use of RBC genotyping as part of a recruitment strategy has not been adequately evaluated and could represent a novel mechanism for garnering public interest in blood donation.

\section{Transfusion Strategies}

Although 'perfect match' strategies for all transfusions before alloimmunization occurs is the idealized way to prevent alloimmunization, a program such as this is of high in cost and may make the blood supply limited [19]. In the interim, there are some additional strategies that can be considered to prevent alloimmunization of high-risk minority patients. RhD- or C- units can be considered for patients at risk of developing anti-D or anti-C upon exposure to conventional $\mathrm{RhD}$ or $\mathrm{RhC}$ antigens because their genotype reveals variant $D$ or $C$ alleles that are expected to express 
partial D or C antigens. As understanding of which Rh variants are the most immunogenic continues to improve, patients will be better able to be triaged into higher risk categories that require this type of antigen matching. Providing D- units, aside from using an already limited supply blood product, however has the additional drawback of likely coming from a Caucasian donor where there is the increased risk of exposure to disparate antigens. Extended matching for $\mathrm{Jk}^{\mathrm{b}}, \mathrm{Fy}^{\mathrm{a}}$, and $\mathrm{S}$ could additionally be considered in these cases. In addition, in patients with variant alleles and multiple antibodies requiring the selection of blood products from predominantly African American donors, Rh genotyping of donors may be indicated. Low prevalence and $\mathrm{Rh}$ variants identified in blood donors should be taken into account in the highest-risk patient populations [5].

Genotyping of patients who are destined for chronic transfusion, such as SCD patients of African descent, can support transfusion decision-making. For instance, patients with the DIIIaCE(4-7)-D allele, in which RHCE exons 4-7 have replaced the corresponding regions in the $R H D$, will serologically type as Dand $\mathrm{C}+$ but are at risk of developing anti-C antibodies when exposed to the conventional $\mathrm{C}$ antigen. It is recommended that these patients be transfused with $\mathrm{C}-\mathrm{RBCs}$ [12]. In addition, the $\mathrm{Fy}(\mathrm{a}-\mathrm{b}-)$ phenotype is common in populations of African ancestry but only about $17 \%$ of donors in the USA are Fy(b-). The majority of African descent individuals who are serologically negative for $\mathrm{Fy}^{\mathrm{b}}$ also have a silencing $\mathrm{Fy}^{\mathrm{b}}$ mutation [17]. This mutation (-67t>c, GATA) prevents only their RBCs from expressing $\mathrm{Fy}^{\mathrm{b}}$. Other cells in their body will express $\mathrm{Fy}^{\mathrm{b}}$ making them low risk for developing an antibody to $\mathrm{Fy}^{\mathrm{b}}$. When patients with GATA mutations are allowed to receive $F y(b+)$ units, it more than doubles the ability to find available units, even for highly restrictive matching [17].

\section{Conclusions}

Advances in transfusion medicine have determined that the causes of RBC allosensitization are multi-factorial. A foundational risk factor for alloimmunization is RBC antigenic differences between the patient and donor; this can be more pronounced in patients with minority ancestral backgrounds. Blood collectors' mission is to ensure access to blood products for all patients. Thus, recruitment and typing strategies that support minority patient populations have become increasingly important. Genotyping of patients and donors has led to a greater understanding of causes for persistent alloimmunization in patients with SCD, and has the potential to become an invaluable tool to improve outcomes and clinical management in all minority populations. The feasibility, practicality, and cost-effectiveness of implementing risk-based algorithms for transfusion matching should continue to be evaluated as technology improves and the inventories of genotyped donors increases.

\section{Disclosure Statement}

Dr Delaney has received honoraria from Grifols. Dr Khan has no conflicts to disclose.

\section{References}

1 Yazer MH, Vassallo R, Delaney M, Germain M, Karafin MS, Sayers M, et al: Trends in age and red blood cell donation habits among several racial/ethnic minority groups in the United States. Transfusion 2017;57:1644-1655.

2 Yazer MH, Delaney M, Germain M, Karafin MS, Sayers M, Vassallo R, et al: Trends in US minority red blood cell unit donations. Transfusion 2017;57:1226-1234.

3 Josephson CD, Su LL, Hillyer KL, Hillyer CD: Transfusion in the patient with sickle cell disease: a critical review of the literature and transfusion guidelines. Transfus Med Rev 2007;21:118-133.

$\checkmark 4$ Ngoma AM, Mutombo PB, Ikeda K, Nollet KE, Natukunda B, Ohto H: Red blood cell alloimmunization in transfused patients in sub-Saharan Africa: a systematic review and meta-analysis. Transfus Apher Sci 2016;54:296-302.

5 Chou ST, Jackson T, Vege S, Smith-Whitley K, Friedman DF, Westhoff CM: High prevalence of red blood cell alloimmunization in sickle cell disease despite transfusion from Rh-matched minority donors. Blood 2013;122:1062-1071.

6 6 Delaney M, Wendel S, Bercovitz RS, Cid J, Cohn C, Dunbar NM, et al: Transfusion reactions: prevention, diagnosis, and treatment. Lancet 2016;388:2825-2836.

7 Win N, New H, Lee E, de la Fuente J: Hyperhemolysis syndrome in sickle cell disease: case report (recurrent episode) and literature review. Transfusion 2008;48: 1231-1238.
8 Mwesigwa S, Moulds JM, Chen A, Flanagan J, Sheehan VA, George A, et al: Whole-exome sequencing of sickle cell disease patients with hyperhemolysis syndrome suggests a role for rare variation in disease predisposition. Transfusion 2018;58:726-735.

9 Schonewille H, Haak HL, van Zijl AM: RBC antibody persistence. Transfusion 2000;40:1127-1131.

10 Tormey CA, Stack G: The persistence and evanescence of blood group alloantibodies in men. Transfusion 2009;49:505-512.

11 Food and Drug Administration: Transfusion/Donation Fatalities. Available at www.fda.gov/BiologicsBloodVaccines/SafetyAvailability/ReportaProblem/ TransfusionDonationFatalities/default.htm (last accessed July 9, 2018).

12 Chou ST, Westhoff CM: Application of genomics for transfusion therapy in sickle cell anemia. Blood Cells Mol Dis 2017;67:148-154.

13 Reid ME, Halter Hipsky C, Hue-Roye K, Hoppe C: Genomic analyses of $\mathrm{RH}$ alleles to improve transfusion therapy in patients with sickle cell disease. Blood Cells Mol Dis 2014;52:195-202.

14 Patel SR, Bennett A, Girard-Pierce K, Maier CL, Chonat S, Arthur CM, et al: Recipient priming to one RBC alloantigen directly enhances subsequent alloimmunization in mice. Blood Adv 2018;2:105-115.

15 Higgins JM, Sloan SR: Stochastic modeling of human RBC alloimmunization: evidence for a distinct population of immunologic responders. Blood 2008;112: 2546-2553.
6 Sippert Emilia Ângela, Visentainer Jeane Eliete Laguila, Alves Hugo Vicentin, Rodrigues Camila, Gilli Simone Cristina Olenscki, Addas-Carvalho Marcelo, et al: Red blood cell alloimmunization in patients with sickle cell disease: correlation with HLA and cytokine gene polymorphisms. Transfusion 2016;57:379-389.

17 Wilkinson K, Harris S, Gaur P, Haile A, Armour R, Teramura G, et al: Molecular blood typing augments serologic testing and allows for enhanced matching of red blood cells for transfusion in patients with sickle cell disease. Transfusion 2012;52:381-388.

18 Castro O, Sandler SG, Houston-Yu P, Rana S: Predicting the effect of transfusing only phenotype-matched RBCs to patients with sickle cell disease: theoretical and practical implications. Transfusion 2002;42:684690

19 Putzulu R, Piccirillo N, Orlando N, Massini G, Maresca M, Scavone F, et al: The role of molecular typing and perfect match transfusion in sickle cell disease and thalassaemia: an innovative transfusion strategy. Transfus Apher Sci 2017;56:234-237.

20 Svensson AM, Delaney M: Considerations of red blood cell molecular testing in transfusion medicine. Expert Rev Mol Diagn 2015;15:1455-1464.

21 International Society of Blood Transfusion: Red Cell Immunogenetics and Blood Group Terminology. Available from: www.isbtweb.org/working-parties/redcell-immunogenetics-and-blood-group-terminology/ (last accessed July 9, 2018). 
22 Daniels G: Variants of RhD- current testing and clinical consequences. Br J Haematol 2013;161:461-470.

23 Gaspardi AC, Sippert EA, Macedo MD de, Pellegrino J Jr, Costa FF, et al: Clinically relevant RHD-CE genotypes in patients with sickle cell disease and in African Brazilian donors. Blood Transfus 2016;14:449.

24 Wagner FF, Ladewig B, Flegel WA: The RHCE allele ceRT: D epitope 6 expression does not require D-specific amino acids. Transfusion 2003;43:1248-1254

25 Chou ST, Westhoff CM: Molecular biology of the Rh system: clinical considerations for transfusion in sickle cell disease. ASH Educ Program Book 2009;2009:178184.

26 National Heart, Lung, and Blood Institute: EvidenceBased Management of Sickle Cell Disease: Expert Panel Report, 2014|National Heart, Lung, and Blood Institute (NHLBI). Available at www.nhlbi.nih.gov/ health-topics/evidence-based-management-sickle-celldisease (last accessed July 9, 2018).

27 Chou ST, Fasano RM: Management of patients with sickle cell disease using transfusion therapy: guidelines and complications. Hematol Oncol Clin North Am 2016;30:591-608.
28 Yee MEM, Josephson CD, Winkler AM, Webb J, Luban NLC, Leong T, et al: Red blood cell minor antigen mismatches during chronic transfusion therapy for sickle cell anemia. Transfusion 2017;57:2738-2746.

29 Natukunda B, Schonewille H, Ndugwa C, Brand A: Red blood cell alloimmunization in sickle cell disease patients in Uganda. Transfusion 2010;50:20-25.

30 Olujohungbe A, Hambleton I, Stephens L, Serjeant B, Serjeant G: Red cell antibodies in patients with homozygous sickle cell disease: a comparison of patients in Jamaica and the United Kingdom. Br J Haematol 2001;113:661-665.

31 Flegel WA, Gottschall JL, Denomme GA: Implementing mass-scale red cell genotyping at a blood center. Transfusion 2015;55:2610-2615; quiz 2609.

32 Denomme GA, Anani WQ, Avent ND, Bein G, Briggs LB, Lapadat RC, et al: Red cell genotyping precision medicine: a conference summary. Ther Adv Hematol 2017;8:277-291.
33 Prisco Arnoni C, Guilhem Muniz J, de Paula Vendrame TA, de Medeiros Person R, Roche Moreira Latini F, Castilho L: RHCE variants inherited with altered RHD alleles in Brazilian blood donors. Transfus Med 2016;26:285-290.

34 Shaz BH, Hillyer CD: Minority donation in the United States: challenges and needs. Curr Opin Hematol 2010; 17:544-549.

5 Cameron AM, Massie AB, Alexander CE, Stewart B, Montgomery RA, Benavides NR, et al: Social media and organ donor registration: the Facebook effect. Am J Transplant 2013;13:2059-2065.

36 Frye V, Caltabiano M, Kessler DA, Schaffler H, Reboza $\mathrm{M}$, Hillyer $\mathrm{CD}$, et al: Evaluating a program to increase blood donation among racial and ethnic minority communities in New York City. Transfusion 2014;54: 3061-3067. 